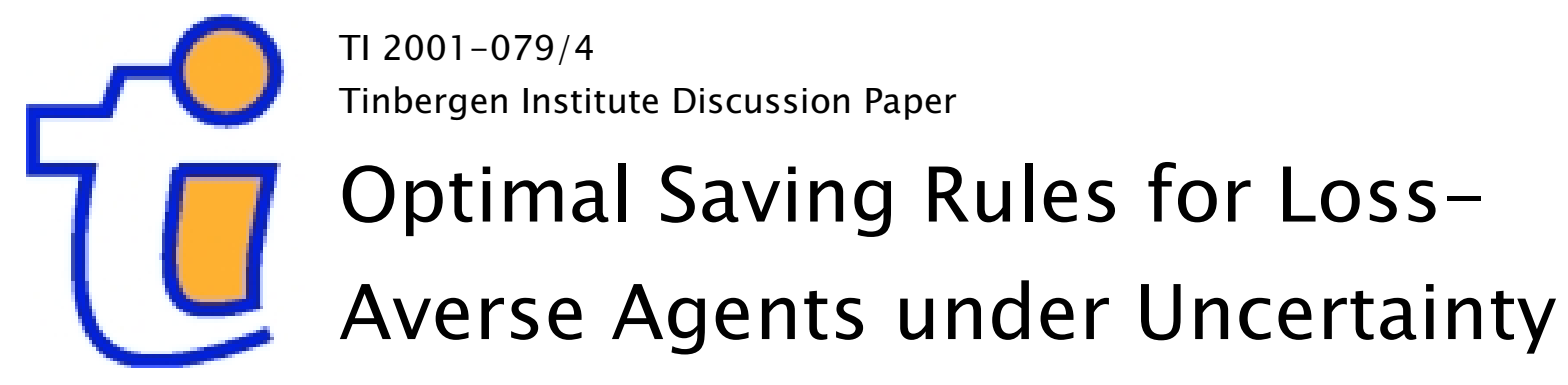

Arjen H. Siegmann

Department of Finance, Faculty of Economics and Business Administration, Vrije Universiteit Amsterdam 
Tinbergen Institute

The Tinbergen Institute is the institute for economic research of the Erasmus Universiteit Rotterdam, Universiteit van Amsterdam and

Vrije Universiteit Amsterdam.

Tinbergen I nstitute Amsterdam

Keizersgracht 482

1017 EG Amsterdam

The Netherlands

Tel.: +31.(0)20.5513500

Fax: $\quad+31 .(0) 20.5513555$

Tinbergen Institute Rotterdam

Burg. Oudlaan 50

3062 PA Rotterdam

The Netherlands

Tel.: $\quad+31 .(0) 10.4088900$

Fax: $\quad+31 .(0) 10.4089031$

Most TI discussion papers can be downloaded at

http://www.tinbergen.nl 


\title{
Optimal saving rules for loss-averse agents under uncertainty
}

\author{
Arjen H. Siegmann \\ Department of Finance, Faculty of Economics, Vrije Universiteit Amsterdam, \\ De Boelelaan 1105, NL-1081 HV, the Netherlands, siegmann@tinbergen.nl
}

This version: June 15, 2001

\begin{abstract}
Most empirical studies assume only monotonic preferences for households. Behavioral research however provides substantial evidence that preferences for wealth are measured relative to a reference point. In this paper we introduce and solve a two-period consumption and savings model for a loss-averse agent who measures utility from consumption relative to a benchmark level. The solution is given as a parametric decision rule with one unknown parameter that depends on the distribution of the return on saving. We find non-linearity in the fraction of wealth saved, where the specific saving pattern depends on the sign of the real return on savings. The amount of saving is nondecreasing in initial wealth and the riskiness of the return distribution.
\end{abstract}

Keywords: Consumption and savings, behavioral value function, loss aversion

JEL classification: D1, D8, D9, E2

\section{Introduction}

In the past decade, there has been an increasing interest in the behavioral underpinning of standard utility theory. Since the work of Kahneman and Tversky (1979) it has become clear that the standard utility functions, e.g. CRRA, CARA, that had been used for their ease of use do not reflect actual preferences of individuals. Kahneman and Tversky (1979) showed that when people are faced with a gamble that delivers a range of (uncertain) outcomes, (i) they are not expected utility maximizers, and (ii) they evaluate pay-offs relative to a reference point. The former can be modeled by introducing prospective utility as an alternative to expected utility, boiling down to transforming the probability 
of each outcome to a subjective probability. The second phenomenon is modeled by using a value function that is convex for losses and concave for gains. In this paper we concentrate on the second, using a simplified value function to model household saving behavior.

In analyzing the results we specifically look at the relation between savings and wealth. As the model includes a benchmark level of consumption, we come quite naturally to a notion of precautionary savings. Precautionary savings is seen as a motive to explain consumption patterns in empirical studies, as in Caballero (1990), Normandin (1994), and Normandin (1997). Another issue is the way in which uncertainty influences savings. Aizenman (1998) shows that disappointment aversion induces a positive relation between uncertainty and the size of a buffer stock. As we have in the current model the explicit decision rules, we are able to trace the relation between uncertainty and savings.

\section{The model}

We consider an agent with current wealth $w$ who lives two periods and has to decide on his current saving $s$. His first and second period budget constraints are

$$
\begin{aligned}
& c_{1}=w-s \\
& c_{2}=s \cdot(1+r),
\end{aligned}
$$

where $c_{i} \geq 0$ denotes consumption over period $i(i=1,2)$, and $r$ the real return on investment. The return $r$ has an arbitrary absolute-continuous probability distribution function $G(\cdot)$ with support $(-1, \infty)$. It represents the real return on investment, which can be a simple savings account, a portfolio of stocks, etc. Having an arbitrary probability distribution $G(\cdot)$ constrasts with Aizenman (1998) and Bowman et al. (1999), who also examine loss-aversion but restrict uncertainty in income to a binomial type of probability distribution.

We assume that the agent or household has a per-period loss-averse utility function

$$
u\left(c_{i}, b\right)=c_{i}-\lambda \cdot\left(b-c_{i}\right)^{+},
$$

where $b$ is a fixed benchmark level of consumption, $\lambda$ is a loss aversion parameter, and $(y)^{+}$ is used to denote the maximum of 0 and $y$. The utility function in (3) incorporates the 
main features of Kahneman and Tversky's value function, as it measures utility relative to a reference point, and treating positive and negative deviations from the reference point asymmetrically. The penalty parameter $\lambda$ represents the extra marginal costs of not achieving a desired consumption level. If borrowing is possible, $\lambda$ can be thought of as the (expensive) borrowing rate on credit card loans for example. The utility function in (3) has been used before by Benartzi and Thaler (1995) in an attempt to explain the equity premium puzzle. It is also the piecewise-linear equivalent of the one used by Aizenman (1998), where $\lambda$ is the disappointment aversion rate and $b$ the certainty equivalent consumption. Finally, Bowman et al. (1999) derive axiomatic results for lossaverse utility functions of which (3) is a special case.

With (3) as the instantaneous utility function leaving out time-discounting, the agent determines saving through the following maximization problem

$$
\max _{c_{1}} c_{1}-\lambda \cdot\left(b-c_{1}\right)^{+}+\mathbb{E}\left(c_{2}\right)-\lambda \cdot \mathbb{E}\left(b-c_{2}\right)^{+},
$$

subject to budget constraints (1) and (2). Although we are interested in the optimal saving policy, we have formulated the problem to be in line with the traditional formulation, i.e. maximizing over consumption.

\section{Results}

The main results in this paper follow from the following theorem.

Theorem 3.1 If the solution to (4) is bounded, it is given by

$$
c_{1}^{*}= \begin{cases}\min \left(b, w-b /\left(1+r_{g}^{*}\right)\right) & \text { if } \mathbb{E}(r) \geq 0, \\ \max \left(b, w-b /\left(1+r_{l}^{*}\right)\right) & \text { if } \mathbb{E}(r)<0,\end{cases}
$$

where $r_{g}^{*}, r_{l}^{*}$ are the two $\bar{r} s$ that solve

$$
\mathbb{E}[r]+\lambda \int_{-1}^{\bar{r}}(1+r) d G=I_{\{\mathbb{E}[r]>0\}} \cdot \lambda
$$

and $I_{\{A\}}$ is defined as the indicator function with respect to the event $A . r_{g}^{*}$ solves (6) for $\mathbb{E}[r] \geq 0$ and $r_{l}^{*}$ for $\mathbb{E}[r]<0$. In addition to equation $(5), c_{1}^{*}$ is restricted to lie between 0 and $w$. 
Proof: See appendix.

Theorem 1 shows that we can derive parametric rules for the optimal consumption fraction. As equation (5) shows, the saving rule differs for positive and negative real returns. The case $\mathbb{E}[r]<0$, corresponds to a situation where the expected positive (nominal) return on investments is smaller than the combined effect of inflation and timediscounting. This situation can occur in economies with hyperinflation for example.

The restriction that $c_{1}^{*}$ lie between 0 and $w$ follows from the imposed nonnegativity of $c_{1}$ and $c_{2}$.

Given the optimal consumption $c_{1}^{*}$ in (5), rewriting $s^{*}=w-c_{1}^{*}$ gives an expression for the optimal amount of savings. It should lie between 0 and $w$, given by

$$
s^{*}= \begin{cases}w-\min \left(b, w-b /\left(1+r_{g}^{*}\right)\right) & \mathbb{E}[r] \geq 0, \\ \min \left([w-b]^{+}, b /\left(1+r_{l}^{*}\right)\right) & \mathbb{E}[r]<0 .\end{cases}
$$

Whereas other studies like Bowman et al. (1999), Aizenman (1998) and Carroll (1998) have derived axiomatic or numerical results for loss-averse models with uncertainty, (7) specifies a parametric relation for optimal saving. The expression in (7) leads to a number of interesting results.

First, observe that the derivative of (7) with respect to $w$ is either 0 or 1 , implying that saving is nondecreasing in wealth. This can be explained by the loss-aversion with respect to period 2 consumption: even if the return on saving is negative, the punishment on period 2 shortfall outweighs the expected money-loss on saving. Note that this also holds when savings is either 0 or $w$, as in this case the derivative is 0 .

Second, (7) reveals the relation between the return distribution $G(\cdot)$, through the values of $r_{g}^{*}$ and $r_{l}^{*}$, and the amount of saving. Carroll (1998) shows that in theoretical models of consumption and saving there can be multiple measures of income uncertainty. This implies that for many of these models it is not trivial to give the relation between saving and uncertainty. In the model presented here, however, we do not have this problem, as the loss-averse utility function in (3) gives an explicit measure of the relevant uncertainty, i.e. risk, which is the expected shortfall below the benchmark level $b$. Moreover, from Theorem 1 it follows that for a given amount of savings, the riskiness of the investment return influences saving unambiguously. To see this, observe from equation (6) that if the 
probability mass below $\bar{r}$ increases, $\bar{r}$ itself should decrease to satisfy the equation. As the $r^{*}$ s have a nonnegative effect on optimal savings through equation (7) it follows that an increase in the riskiness of period 2 wealth has a nondecreasing effect on savings. This means that precautionary saving, i.e. saving to build up a buffer against future risk, is a motive for a loss averse agent as in our model, regardless of the expected return on saving. It corroborates the result by Aizenman (1998), who finds that for disappointment averse (i.e. loss-averse) developing countries, buffer stocks are efficient, with size increasing in the volatility of second period income.

Finally, we derive the optimal fraction of wealth saved. Considering this quantity is relevant because most empirical studies into saving behavior consider the fraction of savings to income, as saving behavior is otherwise not comparable between households with different wealth levels. Dividing left- and right-hand side of equation (7), the savings fraction is restricted to lie between 0 and 1 , given by

$$
(s / w)^{*}= \begin{cases}1-\min \left\{b / w, 1-\frac{b}{w \cdot\left(1+r_{g}^{*}\right)}\right\} & \mathbb{E}[r] \geq 0, \\ \min \left\{1-b / w, \frac{b}{w \cdot\left(1+r_{l}^{*}\right)}\right\} & \mathbb{E}[r]<0 .\end{cases}
$$

Browning and Lusardi (1996) note on the motives for household saving that "there is a widespread feeling that the wealthy have different motives to save from the less wealthy." This suggestion is explicitly confirmed and illustrated through (8). In discussing the difference in savings between the poor and the wealthy, we need to make an extra distinction between economies with a positive and a negative real return. This distinction, which was already present in the solution in (5), has not been observed before.

To start with high return economies, we can define $b+b /\left(1+r_{g}^{*}\right)$ as the threshold wealth that distinghuishes rich from poor households. For agents with wealth below the threshold, relative saving is a decreasing, convex function of wealth. Above the threshold, the fraction of savings it is increasing and concave.

For low-return economies the threshold lies at $b+b /\left(1+r_{l}^{*}\right)$. Below the threshold relative saving is an increasing, concave function of wealth. Above threshold it is a decreasing, convex function of wealth.

To illustrate the difference in the savings fraction for the two types of households and two types of economies, we graph the optimal saving rules for a representative set of parameters. With $b=60$ we have two return distributions, one with a positive and one 


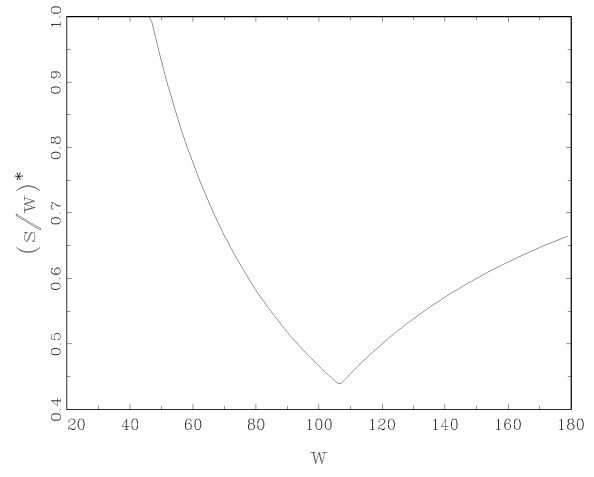

(a)

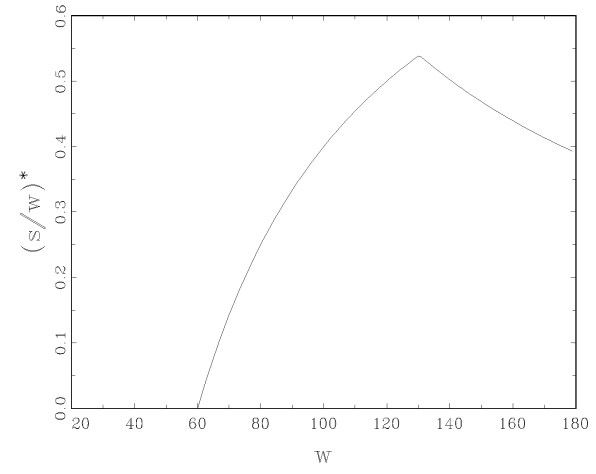

(b)

Figure 1: The optimal fraction of wealth saved, $s_{0}^{*}$, as a function of wealth at time $0, w$. For both graphs $\lambda=1, b=60$. In (a) $1+r$ is distributed lognormal with $\mu=0.085, \sigma=0.16$, so $\mathbb{E}[r]>0$. In (b) $G$ is also lognormal, $\mu=-0.04, \sigma=0.07$, so $\mathbb{E}[r]<0$.

with a negative return. Starting with the case of a positive expected return, we assume $1+r$ follows a lognormal distribution with $\mu=0.085, \sigma=0.17$, being a typical (stylized) stock return distribution. For the negative expected return, we have $\mu=-0.04$ and $\sigma=0.07$, corresponding to a less risky return distribution, but with a negative expected return. This can represent the case of, say, an investment in a bond portfolio in an economy with high inflation. The optimal fractions saved are in Figure 1.

In Figure 1 one can observe that for wealth equal to the benchmark, the savings fraction is at its maximum or minimum, depending on whether the expected return is positive or negative, respectively. When wealth is not at this extreme, Figure 1(a) shows that for poor households a higher initial wealth results in a smaller fraction of wealth saved. For wealthy households the opposite effect holds: an increase in wealth results in increased relative savings. From the list of motives given by Browning and Lusardi (1996), the behavior of the poor households could be well explained by the motive of precautionairy savings. As wealth increases, less money needs to be saved to reach the time 1 benchmark $b$. For wealthy households, the savings pattern is explained by the motive of bequest savings. As wealth increases, more money can be saved to build up a 
possible time 1 fortune.

For Figure 1(b) the situation is exactly the opposite. The fraction of wealth saved is increasing in wealth for poor households and decreasing in wealth for rich households.

In a setting with loss aversion, Bowman et al. (1999) focus on the effects of shocks to income on consumption. They find evidence that gains and losses have an assymetric impact on household consumption and thus saving behavior. In the current model we can conclude that shocks in wealth have a disctinctly different impact on saving behavior, depending on whether the household is relatively poor or wealthy. To see this, consider a household that has a benchmark and current wealth such that saving is minimal/maximal for a positive/negative expected return. The assymetry in the reaction to a change in wealth is caused by the difference in slope between the left-hand and right-hand side of Figure 1.

\section{Conclusion}

In this paper we have we have incorporated a linear version of Kahneman \& Tversky's value function into a standard consumption-savings framework. The formulation makes it possible to derive explicit decision rules for optimal saving. From these rules we concluded that savings is increasing in wealth and in risk, the latter being the probability mass in the left tail of the return distribution. Furthermore, a remarkable result is that saving policies are different for poor and rich households and different types of economies.

If the behavioral value function found by Kahneman and Tversky (1979) is indeed a consistent phenomenon, our results provide strong indications that savings behavior in consumer panel data can be highly non-monotonic in wealth.

\section{Appendix: Proof of Theorem 1}

We start with a reformulation of the optimization problem in (4):

$$
\begin{array}{ll}
\max _{c_{1}} & c_{1}-\lambda \cdot\left(b-c_{1}\right)^{+}+\mathbb{E}[s \cdot(1+r)]-\lambda \cdot \mathbb{E}[b-s \cdot(1+r)]^{+}, \\
\text {s.t. } & s=w-c_{1} .
\end{array}
$$


Denote the value of the objective function (A1) for a fixed value of $c_{1}$ by $f\left(c_{1}\right)$. We have as a first order condition:

$$
\mathbb{E}\left[\frac{d f}{d c_{1}}\right]=0
$$

where

$$
\mathbb{E}\left[\frac{d f}{d c_{1}}\right]= \begin{cases}1+\lambda-\mathbb{E}[1+r]-\lambda \cdot \int_{-1}^{\bar{r}}(1+r) d G & \text { if } c_{1} \leq b, \\ 1-\mathbb{E}[1+r]-\lambda \cdot \int_{-1}^{\bar{r}}(1+r) d G & \text { if } c_{1}>b\end{cases}
$$

and $\bar{r}$ is defined by

$$
\bar{r}=\frac{b}{w-c_{1}}-1
$$

i.e., the threshold return that gives $b-s \cdot(1+r)=0$.

The second order condition to problem (A1) is given by

$$
\mathbb{E}\left[\frac{d^{2} f}{d c_{1}^{2}}\right]=-\lambda \cdot \frac{b^{2}}{\left(w-c_{1}\right)^{3}} \cdot g(\bar{r})<0,
$$

where $g(\cdot)$ is the density function of the return $r . g(\cdot)$ is nonnegative by definition, so the second order condition ensures that any $c_{1}<w$ that satisfies (A4) is optimal.

As $G(\cdot)$ has support on $(-1, \infty)$, the derivative for $c_{1} \leq b$ in (A4) is strictly decreasing in $\bar{r}$. For $\mathbb{E}[r] \geq 0$ it follows from (A4) that $c_{1}^{*} \leq b$, as for $c_{1}>b$ the derivative with respect to $c_{1}$ is negative for all $\bar{r}$. Hence, the appropriate derivative in the case $\mathbb{E}[r] \geq 0$ is the first line in (A4). This includes the case $c_{1}=0$, when either $\mathbb{E}[r]>\lambda$ or $w \leq b /\left(1+r_{g}^{*}\right)$.

If $\mathbb{E}[r]<0$, it follows from (A4) that $c_{1}^{*}>b$, as for $c_{1} \leq b$ the derivative with respect to $c_{1}$ is strictly positive for all $\bar{r}$. Hence, the appropriate derivative is the second line in equation (A4).

From (A4) and using $\lambda>0$, it is straightforward to formulate the conditions under which (A1) - (A2) has a bounded optimal solution. If $c_{1}^{*}>b$ we require $(1+\lambda) \cdot \mathbb{E}[1+r]>1$. In case $c_{1}^{*} \leq b$, the condition $\mathbb{E}[r]<\lambda$ is sufficient.

If the solution to (A1) is bounded, the solution is determined by the $r_{g}^{*}$ and $r_{l}^{*}$ that follow from putting the appriopriate derivative in (A4) to zero and solving for $\bar{r}$. As there is no other term including $c_{1}$ in (A4), the optimal $c_{1}^{*}$ follows directly from the calculated bounds on $c_{1}^{*}$ and the definition of $\bar{r}$. Within the bounds, it is given by

$$
c_{1}^{*}= \begin{cases}\min \left(b, w-b /\left(1+r_{g}^{*}\right)\right) & \text { if } \mathbb{E}[r] \geq 0, \\ \max \left(b, w-b /\left(1+r_{l}^{*}\right)\right) & \text { if } \mathbb{E}[r]<0 .\end{cases}
$$




\section{References}

Aizenman, J. (1998). Buffer stocks and precautionary savings with loss aversion. Journal of International Money and Finance 17, 931-947.

Benartzi, S. and R. H. Thaler (1995). Myopic loss aversion and the equity premium puzzle. Quarterly Journal of Economics 110, 73-92.

Bowman, D., D. Minehart, and M. Rabin (1999). Loss aversion in a consumptionsavings model. Journal of Economic Behavior ES Organization 38, 155-178.

Browning, M. and A. Lusardi (1996, December). Household saving: Micro theories and micro facts. Journal of Economic Literature 34, 1797 - 1855.

Caballero, R. J. (1990). Consumption puzzles and precautionary savings. Journal of monetary economics 25, 113-136.

Carroll, C. D. (1998). How important is precautionary saving? Review of Economics and Statistics 80(3), 410-419.

Kahneman, D. and A. Tversky (1979, March). Prospect theory: An analysis of decision under risk. Econometrica 47, 263-291.

Normandin, M. (1994, April). Precautionary saving: An explanation for excess sensitivity of consumption. Journal of Business and economics statistics 12(2), 205-219.

Normandin, M. (1997). Precautionary saving and the deaton paradox. Applied Economics Letters 4, 187-190. 